\title{
Structural Health Monitoring by Recursive Bayesian Filtering
}

\author{
Yangbo Chen, Ph.D., M.ASCE ${ }^{1}$; and Maria Q. Feng, F.ASCE ${ }^{2}$
}

\begin{abstract}
A new vision of structural health monitoring (SHM) is presented, in which the ultimate goal of SHM is not limited to damage identification, but to describe the structure by a probabilistic model, whose parameters and uncertainty are periodically updated using measured data in a recursive Bayesian filtering (RBF) approach. Such a model of a structure is essential in evaluating its current condition and predicting its future performance in a probabilistic context. RBF is conventionally implemented by the extended Kalman filter, which suffers from its intrinsic drawbacks. Recent progress on high-fidelity propagation of a probability distribution through nonlinear functions has revived RBF as a promising tool for SHM. The central difference filter, as an example of the new versions of RBF, is implemented in this study, with the adaptation of a convergence and consistency improvement technique. Two numerical examples are presented to demonstrate the superior capacity of RBF for a SHM purpose. The proposed method is also validated by large-scale shake table tests on a reinforced concrete two-span three-bent bridge specimen.
\end{abstract}

DOI: 10.1061/(ASCE)0733-9399(2009)135:4(231)

CE Database subject headings: Bayesian analysis; Filters; Monitoring; Assessments; Vibration; Identification; Structural analysis.

\section{Introduction}

Recently, structural health monitoring (SHM) has been a subject intensively investigated. As a branch of the wide-ranging efforts of SHM, many researchers seek to measure the structural vibration behaviors (dynamic responses of a structure with or without measuring the exerting excitations), and infer from the vibration data the level of structural integrity. A thorough literature review on the vibration-based SHM was first presented by Doebling et al. (1996), summarizing hundreds of publications up to 1995. A fourlevel hierarchy, namely, (1) detecting the existence of damage; (2) locating damaged portions; (3) evaluating the severity of damage; and (4) predicting its future consequences, was cited and defined as the goals of SHM. Recently, an updated review of the state was presented by Sohn et al. (2003), summarizing publications from 1996 to 2001. This review interprets vibration-based SHM following a statistical pattern recognition paradigm, consisting of a four-part process: (1) operational evaluation; (2) data acquisition, fusion, and cleansing; (3) feature extraction and information condensation; and (4) statistical model development for feature discrimination. In this paradigm, features that are believed damage sensitive are extracted from vibration data, and a pattern recognition procedure is employed to classify the feature vectors in order to determine the existence, location, and severity of structural damage. While the important role of statistical methods in SHM was recognized, the ultimate goal of SHM is still damage identi-

${ }^{1}$ Englekirk Partners Consulting Structural Engineers, Inc., Los Angeles, CA 90018; formerly, Graduate Student, Dept. of Civil and Environmental Engineering, Univ. of California at Irvine, Irvine, CA 92697-2175. E-mail: yangbo.chen@englekirk.com

${ }^{2}$ Professor, Dept. of Civil and Environmental Engineering, Univ. of California, at Irvine, Irvine, CA 92697-2175. E-mail: mfeng@uci.edu

Note. Associate Editor: Erik A. Johnson. Discussion open until September 1, 2009. Separate discussions must be submitted for individual papers. The manuscript for this paper was submitted for review and possible publication on March 30, 2006; approved on April 25, 2008. This paper is part of the Journal of Engineering Mechanics, Vol. 135, No. 4, April 1, 2009. CASCE, ISSN 0733-9399/2009/4-231-242/\$25.00. fication, as was defined in the previous review. In view of the difficulties associated with mathematical models (often referring to finite-element models) of structural systems, especially the difficulty in quantifying the modeling uncertainty and the bias due to modeling errors, the reviewers uphold methods that are not based on mathematical models as more attractive. However, difficulties of non-model-based methods were also recognized, especially in quantifying the severity of damage where a supervised learning mode is usually adopted. Training patterns have to be generated by a mathematical model whose fidelity remains to be verified, because data sets from a damaged real-life structure are seldom documented, and if they do exist, they are not adequate to cover all possible damage scenarios. A sufficient coverage on various scenarios by training patterns, nonetheless, is essential in the supervised learning procedure.

Evident by these reviews and more recent papers (e.g., Bolton et al. 2001; Hera 2004; Koh et al. 2003; Lam et al. 2004; Yang and Lin 2005), despite significant efforts, damage identification by SHM is still a highly challenging problem. When implementing vibration-based SHM to real-life structures, the limitation of sensing capacity (e.g., spatial limitation due to insufficient sensor number or prohibitive positions of instrumentation, and temporal limitation due to insufficient sensor frequency range and excitation bandwidth), and the operational and environmental variation of the structures have significantly increased the difficulties.

Nonetheless, the writers of this paper believe that part of the challenges in SHM can be attributed to a preference among scholars to an inductive, objective, and entirely data-driven methodology. A shift of epistemology from a purely inductive to a deductive-inductive hybrid methodology might help to ease the problem and bring forward useful results. In the deductiveinductive methodology, a priori knowledge, derived either from established theories, engineering experiences, or even subjective postulations, is incorporated in a probabilistic model of the structural system. In this model, the extent of knowledge limitation is represented by the uncertainty of the model structure and parameters. This model is subjected to correction or refinement based on sensor data, by first deducing the expected vibration behaviors from the a priori model, and then comparing them with the sensor 
observations and updating the model in a systematic induction to reconcile the predicted and observed vibration. The advantage of this approach is that gaps of necessary information not provided by sensor data are filled in with the currently available best understanding of the system. Therefore, SHM is no longer merely a mean of nondestructive damage evaluation, but a procedure of information collection to correct or refine the probabilistic model of the structural system so as to diminish, gradually, the system uncertainty.

The above methodology is essentially a Bayesian approach. This vision of SHM can be traced back to Beck (1989), where a Bayesian framework was laid down for structural system identification that selects the most probable model from a class of models based on input/output measurement. Later, Beck and Katafygiotis (1998) formalized this version not only to update the model, but also to assess the uncertainties of the model itself and its predictions. This formulation addresses explicitly the difficult problem in parameter identification: the inherent ill conditioning and nonuniqueness. In the latter case, prediction of structural behaviors is still possible in this framework, using more than one candidate models, but weighting their predictions according to their model a posteriori probability. This was treated by Beck and $\mathrm{Au}$ (2002) using a Markov chain Monte Carlo method. Beck and Yuen (2004) extended the Bayesian framework to address the modeling error issue arising when the "true" system is not within the class of models being examined. The capacity of a dataupdated model to predict the structural responses to future loads, in a probabilistic sense, was utilized to make a connection between SHM results and structural reliability evaluation ( $\mathrm{Pa}-$ padimitriou et al. 2001; Beck and Au 2002). Vanik et al. (2000) treated the variation of modal parameters (frequencies and mode shapes) in a Bayesian framework to set a probabilistic measure of the significance of modal changes. Although damage identification is not the major concern of the model updating procedure, it is also possible if damage can be defined quantitatively in terms of parameter changes (Yuen et al. 2004).

This approach is certainly model dependent. However, it can be argued that models are almost inevitable anyway in structural condition assessment (e.g., in training pattern generation) and in evaluation of current and future performance of a structure. To minimize the disadvantage caused by modeling errors, one shall avoid a deterministic perspective of a model, but instead, shall use a probability to measure the level of modeling uncertainty.

This Bayesian approach for SHM, however, often results in extremely demanding computation in high dimensional spaces. This paper focuses only on a relatively tractable problem, recursive Bayesian filtering (RBF). Operating in a recursive mode, $\mathrm{RBF}$ is suitable for a continuous SHM practice. This paper will review the development of $\mathrm{RBF}$, the obstacles that have limited its successful usage in the past, some recent breakthroughs that can revive this approach for SHM, and a technique that can be incorporated to improve the convergence and estimation consistency. Then, two numerical examples will be presented to demonstrate the use of RBF for SHM purposes. And, finally, this method is validated by data obtained in a large-scale shake table test on a reinforced concrete bridge specimen.

\section{Review on RBF}

The Bayesian theorem provides a way to infer conditional probability $P\left(B_{i} \mid A\right)$ from conditional probability $P\left(A \mid B_{i}\right)$, where events $\left\{B_{i}\right\}, i=1$ to $n$ are a set of mutually exclusive and collectively exhaustive events, by

$$
P\left(B_{i} \mid A\right)=\frac{P\left(A \mid B_{i}\right) P\left(B_{i}\right)}{\sum_{i=1}^{n} P\left(A \mid B_{i}\right) P\left(B_{i}\right)}
$$

If only the relative likelihood of $B_{i}$ is concerned, the denominator in Eq. (1) is simply a constant of normalization.

In a Bayesian point of view, conditional probability $P\left(B_{i} \mid A\right)$, or the a posteriori probability, conveys the entire knowledge about $B_{i}$ after observing the occurrence of event $A$. Based on $P\left(B_{i} \mid A\right)$, one can obtain optimal estimation of $B_{i}$, as well as the uncertainty associated with the estimation. Similarly, $P\left(B_{i}\right)$ conveys the entire a priori knowledge about $B_{i}$, which is subjected to correction or refinement. Conditional probability $P\left(A \mid B_{i}\right)$ represents the procedure of deduction: how likely $A$ is to occur as a result of $B_{i}$. Therefore, a Bayesian framework is a vehicle that implements the deductive-inductive approach outlined previously. When recursively applied to a dynamic system represented by a state-space model, to estimate the current system states based on previous and current observations, the framework is referred to as RBF.

\section{$R B F$}

A state-space model is defined as

$$
\begin{gathered}
\mathbf{X}_{k}=f\left(\mathbf{X}_{k-1}, \mathbf{U}_{k-1}, \mathbf{W}_{k-1} ; \boldsymbol{\theta}\right) \\
\mathbf{Z}_{k}=h\left(\mathbf{X}_{k}, \mathbf{U}_{k}, \mathbf{V}_{k} ; \boldsymbol{\theta}\right)
\end{gathered}
$$

where $\mathbf{X}=$ hidden states; $\mathbf{Z}=$ observations (measurements); $\mathbf{U}=$ deterministic input; $\mathbf{W}=$ process noise; $\mathbf{V}=$ measurement noise; and $k=$ time index. The state transfer function $f$ and observation function $h$ are argumented by parameters $\boldsymbol{\theta}$, which could be dependent on $k$. Applying Eq. (1), the RBF at time $k$ is

$$
p\left(\mathbf{X}_{k} \mid \mathbf{Z}_{1: k}\right)=\frac{p\left(\mathbf{Z}_{k} \mid \mathbf{X}_{k}\right) p\left(\mathbf{X}_{k} \mid \mathbf{Z}_{1: k-1}\right)}{\int p\left(\mathbf{Z}_{k} \mid \mathbf{x}_{k}\right) p\left(\mathbf{x}_{k} \mid \mathbf{Z}_{1: k-1}\right) d \mathbf{x}_{k}}
$$

where $\mathbf{Z}_{1: k}$ denotes the collection of observations from time 1 to $k$. Further, in view of state transfer and observation functions in Eqs. (2) and (3)

$$
p\left(\mathbf{X}_{k} \mid \mathbf{Z}_{1: k}\right)=\frac{p\left(\mathbf{Z}_{k} \mid \mathbf{X}_{k}\right) \int p\left(\mathbf{X}_{k} \mid \mathbf{x}_{k-1}\right) p\left(\mathbf{x}_{k-1} \mid \mathbf{Z}_{1: k-1}\right) d \mathbf{x}_{k-1}}{\iint p\left(\mathbf{Z}_{k} \mid \mathbf{x}_{k}\right) p\left(\mathbf{x}_{k} \mid \mathbf{x}_{k-1}\right) p\left(\mathbf{x}_{k-1} \mid \mathbf{Z}_{1: k-1}\right) d \mathbf{x}_{k-1} d \mathbf{x}_{k}}
$$

Probability density $p\left(\mathbf{X}_{k-1} \mid \mathbf{Z}_{1: k-1}\right)$ represents the knowledge updated using measurements up to time $k-1$. Conditional probabilities $p\left(\mathbf{X}_{k} \mid \mathbf{X}_{k-1}\right)$ and $p\left(\mathbf{Z}_{k} \mid \mathbf{X}_{k}\right)$ represent the deduction procedure by Eqs. (2) and (3), respectively. And $p\left(\mathbf{X}_{k} \mid \mathbf{Z}_{1: k}\right)$ is the a posteriori after incorporating information gained in measurement $\mathbf{Z}_{k}$. This procedure is applied recursively as $k$ increases. At $k=0$, the a priori probability density to initiate RBF is given by $p\left(\mathbf{X}_{0}\right)$.

RBF, as in Eq. (5), is valid for general systems that can be represented by a state-space model. However, it turns out that the deduction through Eqs. (2) and (3), and the integration in Eq. (5), are only tractable when Eqs. (2) and (3) are both linear and noise sources $\mathbf{W}$ and $\mathbf{V}$ are independent Gaussian white processes. In this linear-Gaussian case, it leads to a closed-form solution, the celebrated Kalman filter.

\section{Parameter Estimation: Difficulties and Breakthroughs}

For the SHM purpose, however, structural properties (e.g., element stiffness) are of concern, which usually is represented by 
parameter $\boldsymbol{\theta}$ in functions $f$ and $h$. To trace structure changes or to update the model and its uncertainty, it suffices to estimate $\boldsymbol{\theta}$ and its probability distribution. To this end, $\boldsymbol{\theta}$ is regarded as part of the extended state $\left\{\mathbf{X}_{k}, \boldsymbol{\theta}_{k}\right\}^{T}$ and the state-space model is restructured as

$$
\begin{aligned}
\left\{\mathbf{X}_{k}, \boldsymbol{\theta}_{k}\right\}^{T}= & F\left(\left\{\mathbf{X}_{k-1}, \boldsymbol{\theta}_{k-1}\right\}^{T}, \mathbf{U}_{k-1}, \mathbf{W}_{k-1}\right) \\
\mathbf{Z}_{k} & =H\left(\left\{\mathbf{X}_{k}, \boldsymbol{\theta}_{k}\right\}^{T}, \mathbf{U}_{k}, \mathbf{V}_{k}\right)
\end{aligned}
$$

where Eq. (6) is the result of incorporating Eq. (2) with

$$
\boldsymbol{\theta}_{k}=\boldsymbol{\phi} \boldsymbol{\theta}_{k-1}+\mathbf{W}_{k-1}^{\boldsymbol{\theta}}
$$

and Eq. (7) is Eq. (3) undergoing necessary adjustment. When the structural system is believed not to vary very fast, usually, in Eq. (8) $\boldsymbol{\phi}=\mathbf{I}$, where $\mathbf{I}=$ unit matrix.

Unfortunately, even when $f$ and $h$ are linear with respect to $\mathbf{X}$, $F$ and $H$ usually are no longer linear with respect to $\boldsymbol{\theta}$, nor to the extended state $\{\mathbf{X}, \boldsymbol{\theta}\}^{T}$. Gaussian probability densities, when propagating through nonlinear functions, are no longer Gaussian or tractable, rendering the RBF not to have a close-form solution.

The first solution adopted for nonlinear RBF is the extended Kalman filter (EKF), which approximates the nonlinear $F$ and $H$ by their first-order Taylor expansions about the current expected mean of the extended state. Adoption of the EKF for parameter identification of civil engineering structures was pioneered by Yun and Shinozuka (1980), Shinozuka et al. (1982), and Yun et al. (1989). Since then, numerous investigations on the EKF have been carried out, with different levels of success in solving the identification problem. However, it has been a consensus that the implementation of the EKF is difficult, especially for complicated civil structures (Ghanem and Shinozuka 1995; Shinozuka and Ghanem 1995). Divergence often is observed due to a number of factors, such as the initial guess of the parameters (including mean and variance), estimation of variances of the process and measurement noises, choice of observed response, unidentifiability of the system, and numerical ill conditioning (Chui and Chen 1991; Grewal and Andrews 2001; Hoshiya and Saito 1984; Hoshiya and Sutoh 1993; Koh and See 1994; Oreta and Tanabe 1994; Yun et al. 1989). It has been shown that the major drawback of the EKF is that it employs a local linearization to approximate the propagation of a probability density through a nonlinear system, which can seriously distort the distribution and shift the mean, incorrectly emphasizing the local behavior of the nonlinear functions (Wan and van der Merwe 2001). In recursive operation, such errors accumulate and lead to divergence.

Recently, breakthroughs in high-fidelity probability density propagation in nonlinear systems have been made using concepts based on the importance sampling principles (e.g., Srinivasan 2002). They have revived the RBF approach, with many new versions of RBF proposed, such as the Gaussian sum, grid-based, Monte Carlo particle, unscented Kalman, central difference, and divided difference filters. The last three are uniformly presented as sigma point filters by van der Merwe (2004). Several of these new versions of RBF have been applied to civil engineering problems, especially for SHM applications (Moradkhani et al. 2005; Yoshida and Sato 2002a,b). This study focuses on one of the new RBF methods that compromises the accuracy of the approximation and the computational efficiency, the central difference filter (CDF).

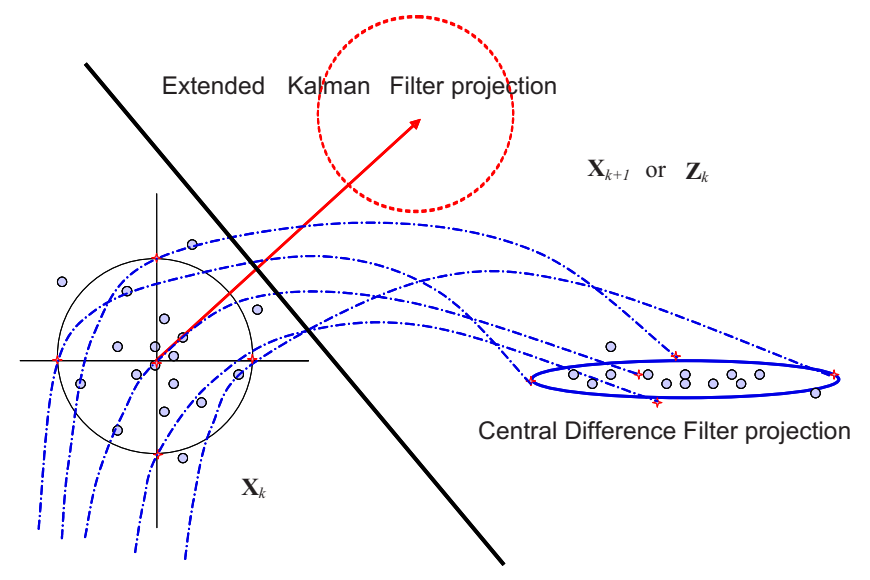

(a) Conceptual Illustration of CDF

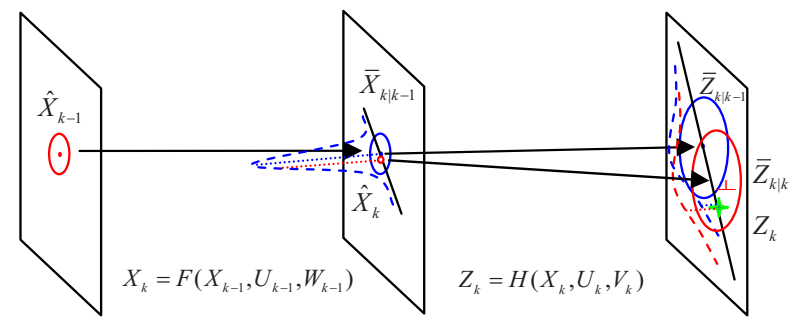

(b) Estimation of $\hat{\mathbf{X}}_{k}$

Fig. 1. Probability density propagation and estimation of $\hat{\mathbf{X}}_{k}$

\section{CDF Algorithm}

The CDF proceeds by weighted statistical linear regression of functions $F$ and $H$. Instead of linearizing the functions at one local point, the CDF selects a limit number of representative points spreading according to the a priori distribution (called the sigma points), propagates each individual point through the nonlinear functions, and obtains the new center and spread of the distribution by linear regression of the points cast forward. [See Fig. 1(a) for an illustration of the concept of CDF.] It is assumed that joint distributions (before/after the nonlinear transformation) are represented adequately by their first and second moments. Propagation of multiple points and the linear regression enhance the capacity of the CDF to capture the nonlinear behavior of the system functions. To elaborate, the $\mathrm{CDF}$ can be implemented as follow (van der Merwe 2004).

If the distribution of $\mathbf{X}_{k-1} \mid \mathbf{Z}_{1: k-1}$ has mean $\hat{\mathbf{X}}_{k-1}$ and variance $\hat{\mathbf{P}}_{k-1}^{\mathbf{X X}}$ (here, for simplicity, $\mathbf{X}_{k}$ represents the extended state $\left\{\mathbf{X}_{k}, \boldsymbol{\theta}_{k}\right\}^{T}$, and process noise $\mathbf{W}_{k-1}$ has mean $\boldsymbol{\mu}_{k-1}^{\mathbf{W}}$ and variance $\mathbf{P}_{k-1}^{\mathbf{W W}}$, let $\operatorname{diag}\left(\hat{\mathbf{P}}_{k-1}^{\mathbf{X X}}, \mathbf{P}_{k-1}^{\mathbf{W W}}\right)$ denote a block diagonal matrix that combines $\hat{\mathbf{P}}_{k-1}^{\mathbf{X X}}$ and $\mathbf{P}_{k-1}^{\mathbf{W W}}$ in such a way that

$$
\operatorname{diag}\left(\hat{\mathbf{P}}_{k-1}^{\mathbf{X X}}, \mathbf{P}_{k-1}^{\mathbf{W W}}\right) \equiv\left[\begin{array}{cc}
\hat{\mathbf{P}}_{k-1}^{\mathbf{X X}} & 0 \\
0 & \mathbf{P}_{k-1}^{\mathbf{W W}}
\end{array}\right]
$$

Sigma points for time update are selected as in Eq. (10), where sigma points are denoted collectively by $\mathbf{S}_{k-1}^{t}$ in matrix format 


$$
\begin{aligned}
\mathbf{S}_{k-1}^{t} \equiv & {\left[\begin{array}{l}
\mathbf{S}_{k-1}^{t \mathbf{X}} \\
\mathbf{S}_{k-1}^{t \mathbf{W}}
\end{array}\right]=\left\{\begin{array}{l}
\hat{\mathbf{X}}_{k-1} \\
\boldsymbol{\mu}_{k-1}^{\mathbf{W}}
\end{array}\right\} \oplus\left[\left|\mathbf{0} \sqrt{3 \cdot \operatorname{diag}\left(\hat{\mathbf{P}}_{k-1}^{\mathbf{X X}}, \mathbf{P}_{k-1}^{\mathbf{W W}}\right)}\right|\right.} \\
& \left.-\sqrt{3 \cdot \operatorname{diag}\left(\hat{\mathbf{P}}_{k-1}^{\mathbf{X X}}, \mathbf{P}_{k-1}^{\mathbf{W W}}\right)}\right]
\end{aligned}
$$

where symbol $\oplus$ means adding a column vector to each column in a matrix and $\sqrt{\mathbf{M}}$ denotes the Cholesky factor of matrix $\mathbf{M}$. Note that $\mathbf{S}_{k-1}^{t}$ is an $\left(n_{s}+n_{p}+n_{W}\right) \times\left(2 n_{s}+2 n_{p}+2 n_{W}+1\right)$ matrix, where $n_{s}=$ dimension of the state vector; $n_{p}=$ dimension of parameter $\boldsymbol{\theta}$; and $n_{W}=$ dimension of the process noise $\mathbf{W}_{k-1}$. And the $i$ th column in $\mathbf{S}_{k-1}^{t}, \mathbf{S}_{k-1}^{t}(i)=i$ th sigma point. For narration convenience, $\mathbf{S}_{k-1}^{t}$ is divided into two blocks: $\mathbf{S}_{k-1}^{t \mathbf{X}}$ denotes the portion related to extended state $\mathbf{X}$ and $\mathbf{S}_{k-1}^{t \mathbf{W}}$ the portion related to process noise $\mathbf{W}$.

The $i$ th sigma point propagates through Eq. (6), resulting in $\mathbf{X}_{k \mid k-1}(i)$

$$
\mathbf{X}_{k \mid k-1}(i)=F\left[\mathbf{S}_{k-1}^{t \mathbf{X}}(i), \mathbf{U}_{k-1}, \mathbf{S}_{k-1}^{t \mathbf{W}}(i)\right]
$$

By regression, the first two moments of distribution of $\mathbf{X}_{k \mid k-1}$ are

$$
\begin{gathered}
\overline{\mathbf{X}}_{k \mid k-1}=\sum_{i=1}^{r} w_{i} \mathbf{X}_{k \mid k-1}(i) \\
\mathbf{P}_{k \mid k-1}^{\mathbf{X X}}=\sum_{i=1}^{r} w_{i}\left[\mathbf{X}_{k \mid k-1}(i)-\overline{\mathbf{X}}_{k \mid k-1}\right]\left[\mathbf{X}_{k \mid k-1}(i)-\overline{\mathbf{X}}_{k \mid k-1}\right]^{T}
\end{gathered}
$$

where $r=2 n_{s}+2 n_{p}+2 n_{W}+1$ and $w_{i}=$ weights determined by importance sampling principles (van der Merwe 2004).

To propagate through Eq. (7), sigma points collected in matrix format $\mathbf{S}_{k-1}^{m}$ for measurement update are selected as in Eq. (14), where notations are similar to those in Eq. (10)

$$
\begin{aligned}
\mathbf{S}_{k}^{m} \equiv & {\left[\begin{array}{c}
\mathbf{S}_{k}^{m \mathbf{X}} \\
\mathbf{S}_{k}^{m \mathbf{V}}
\end{array}\right]=\left\{\begin{array}{c}
\overline{\mathbf{X}}_{k \mid k-1} \\
\boldsymbol{\mu}_{k}^{\mathbf{V}}
\end{array}\right\} \oplus\left[\left|\mathbf{0} \sqrt{3 \cdot \operatorname{diag}\left(\mathbf{P}_{k \mid k-1}^{\mathbf{X X}}, \mathbf{P}_{k}^{\mathbf{V V}}\right)}\right|\right.} \\
& \left.-\sqrt{3 \cdot \operatorname{diag}\left(\mathbf{P}_{k \mid k-1}^{\mathbf{X X}}, \mathbf{P}_{k}^{\mathbf{V V}}\right)}\right]
\end{aligned}
$$

where measurement noise $\mathbf{V}_{k}$, an $n_{V}$ vector, has mean $\boldsymbol{\mu}_{k}^{\mathbf{V}}$ and variance $\mathbf{P}_{k}^{\mathrm{VV}}$. The deduced observation $\mathbf{Z}_{k \mid k-1}(i)$ for the $i$ th point of $\mathbf{S}_{k-1}^{m}, \mathbf{S}_{k-1}^{m}(i)$, is

$$
\mathbf{Z}_{k \mid k-1}(i)=H\left[\mathbf{S}_{k}^{m \mathbf{X}}(i), \mathbf{U}_{k}, \mathbf{S}_{k}^{m \mathbf{V}}(i)\right]
$$

The first two moments of deduced observation $\mathbf{Z}_{k \mid k-1}$ are

$$
\begin{gathered}
\overline{\mathbf{Z}}_{k \mid k-1}=\sum_{i=1}^{l} w_{i} \mathbf{Z}_{k \mid k-1}(i) \\
\mathbf{P}_{k \mid k-1}^{\mathbf{Z Z}}=\sum_{i=1}^{l} w_{i}\left[\mathbf{Z}_{k \mid k-1}(i)-\overline{\mathbf{Z}}_{k \mid k-1}\right]\left[\mathbf{Z}_{k \mid k-1}(i)-\overline{\mathbf{Z}}_{k \mid k-1}\right]^{T}
\end{gathered}
$$

in which $l=2 n_{s}+2 n_{p}+2 n_{V}+1$. And the cross variance is

$$
\mathbf{P}_{k \mid k-1}^{\mathbf{X Z}}=\sum_{i=1}^{l} w_{i}\left[\mathbf{S}_{k}^{m \mathbf{X}}(i)-\overline{\mathbf{X}}_{k \mid k-1}\right]\left[\mathbf{Z}_{k \mid k-1}(i)-\overline{\mathbf{Z}}_{k \mid k-1}\right]^{T}
$$

Now, upon incorporating the information contained in actual observation $\mathbf{Z}_{k}$, the a posterior estimation of the extended state $\mathbf{X}_{k} \mid \mathbf{Z}_{1: k}$ is, in terms of its mean $\hat{\mathbf{X}}_{k}$ and variance $\hat{\mathbf{P}}_{k}^{\mathbf{X X}}$

$$
\hat{\mathbf{X}}_{k}=\overline{\mathbf{X}}_{k \mid k-1}+\mathbf{K}_{k}\left(\mathbf{Z}_{k}-\overline{\mathbf{Z}}_{k \mid k-1}\right)
$$

$$
\hat{\mathbf{P}}_{k}^{\mathbf{X X}}=\mathbf{P}_{k \mid k-1}^{\mathbf{X X}}-\mathbf{K}_{k} \mathbf{P}_{k \mid k-1}^{\mathbf{Z Z}} \mathbf{K}_{k}^{T}
$$

where the Kalman gain $\mathbf{K}_{k}$ is

$$
\mathbf{K}_{k}=\mathbf{P}_{k \mid k-1}^{\mathbf{X Z}}\left(\mathbf{P}_{k \mid k-1}^{\mathbf{Z Z}}\right)^{-1}
$$

The procedure in Eqs. (10)-(21) is recursively performed for an increased $k$.

\section{Technique to Improve Convergence and Consistency}

New versions of RBF generally perform better than the EKF because of a more robust propagation of the probability distribution in nonlinear systems. However, divergence still was observed when applying new RBF for parameter identification (Yoshida and Sato 2002a). Moreover, a phenomenon of undue confidence that has been well documented in EKF applications still is expected even using new RBF. In the EKF, the parameter uncertainty evolution usually suffers when the variance is too small that it has too much trust in the previous identified results, discarding the new observations. The consistency of uncertainty estimation, which is important in SHM practice, is sacrificed. Many cases of divergence and undue confidence are caused by an improper initial guess of either (1) the a priori mean or (2) the a priori variance of the parameters, which remains problematic even if probability distributions are properly propagated. Two techniques were developed by Hoshiya and Saito (1984) and Koh and See (1994), respectively, to address these issues in the EKF context. These techniques can be adapted for the new RBF versions to alleviate the divergence and inconsistent estimation of uncertainty.

To alleviate the divergence in the EKF due to an improper initial mean, a weighted global iteration (WGI) was developed by Hoshiya and Saito (1984), taking advantage of the parameter stability in Eq. (8). In the WGI, the EKF is initiated with $\hat{\mathbf{X}}_{0}$ and $\hat{\mathbf{P}}_{0}^{\mathbf{X X}}$ to obtain estimation of $\hat{\mathbf{X}}_{s}$ and $\hat{\mathbf{P}}_{s}^{\mathbf{X X}}$, where $s=$ time index of the last datum. Then, $\hat{\mathbf{X}}_{0}$ and $\hat{\mathbf{P}}_{0}^{\mathbf{X X}}$ are replaced by $\hat{\mathbf{X}}_{s}$ and $a \hat{\mathbf{P}}_{s}^{\mathbf{X X}}(a>1)$, respectively, and again, the EKF is iterated. This procedure is repeated until the initial value $\hat{\mathbf{X}}_{0}$ becomes almost equal to the final value $\hat{\mathbf{X}}_{s}$. It is essentially a procedure that runs the EKF with various initial means $\hat{\mathbf{X}}_{0}$. The amplification on the variance $\hat{\mathbf{P}}_{s}^{X X}$ allows more freedom to take a random $\hat{\mathbf{X}}_{1}$. This technique has been applied in the EKF context by Oreta and Tanabe (1994) and Yun et al. (1989), and extended to a local iteration for segments of data by Hoshiya and Sutoh (1993). Similar WGI can be applied easily to the CDF or other new RBF methods.

The undue confidence phenomenon could be best understood in a Bayesian framework. For illustration, assuming that all the probability distributions involved can be approximated by multinormal distributions, the a posteriori in Eq. (4) is rewritten as

$$
p\left(\mathbf{X}_{k} \mid \mathbf{Z}_{1: k}\right)=\frac{\Phi\left[\mathbf{Z}_{k} ; H\left(\mathbf{X}_{k}, \mathbf{U}_{k}, \boldsymbol{\mu}_{k}^{\mathbf{V}}\right), \mathbf{P}_{k}^{\mathbf{V V}}\right] \Phi\left(\mathbf{X}_{k} ; \overline{\mathbf{X}}_{k \mid k-1}, \mathbf{P}_{k \mid k-1}^{\mathbf{X X}}\right)}{\Phi\left(\mathbf{Z}_{k} ; \overline{\mathbf{Z}}_{k \mid k-1}, \mathbf{P}_{k \mid k-1}^{\mathbf{Z Z}}\right)}
$$

where $\Phi(\mathbf{x} ; \overline{\mathbf{x}}, \mathbf{P})=$ multinormal probability density function, as shown in Eq. (23) 


$$
\Phi(\mathbf{x} ; \overline{\mathbf{x}}, \mathbf{P})=(2 \pi)^{-n / 2}|\mathbf{P}|^{-1 / 2} \exp \left[-\frac{1}{2}(\mathbf{x}-\overline{\mathbf{x}})^{T} \mathbf{P}^{-1}(\mathbf{x}-\overline{\mathbf{x}})\right]
$$

Note that the exponent is a measure of the distance between $\mathbf{x}$ and $\overline{\mathbf{x}}$ weighted by $\mathbf{P}^{-1} . \Phi(\mathbf{x} ; \overline{\mathbf{x}}, \mathbf{P})$ increases when the distance $\|\mathbf{x}-\overline{\mathbf{x}}\|$ is shortened. With this geometric interpretation, the maximum likelihood estimation of $\hat{\mathbf{X}}_{k}$ maximizing Eq. (22), is a compromise point that minimizes the sum of weighted distances $\left\|\mathbf{Z}_{k}-H\left(\hat{\mathbf{X}}_{k}, \mathbf{U}_{k}, \boldsymbol{\mu}_{k}^{\mathbf{V}}\right)\right\|$ and $\left\|\hat{\mathbf{X}}_{k}-\overline{\mathbf{X}}_{k \mid k-1}\right\|$. When undue confidence is associated with $\overline{\mathbf{X}}_{k \mid k-1}$, i.e., $\mathbf{P}_{k \mid k-1}^{\mathbf{X X}}$ is too small, the heavy weight on $\left\|\hat{\mathbf{X}}_{k}-\overline{\mathbf{X}}_{k \mid k-1}\right\|$ will constrain $\hat{\mathbf{X}}_{k}$ within the close vicinity of $\overline{\mathbf{X}}_{k \mid k-1}$, impairing its ability to adjust to the new observation $\mathbf{Z}_{k}$ [see Fig. 1(b)].

Note that the maximization procedure is independent of the denominator $p\left(\mathbf{Z}_{k} \mid \mathbf{Z}_{1: k-1}\right)$, or $\Phi\left(\mathbf{Z}_{k} ; \overline{\mathbf{Z}}_{k \mid k-1}, \mathbf{P}_{k \mid k-1}^{\mathbf{Z Z}}\right)$, which, nonetheless, carries information to evaluate the consistency of the estimation. $\mathbf{P}_{k \mid k-1}^{\mathbf{Z Z}}$ is the predicted error covariance, but the actual residual $\mathbf{r}_{k}=\mathbf{Z}_{k}-\overline{\mathbf{Z}}_{k \mid k-1}$ is available at all $k$ 's in the filtering procedure. Assuming that $\mathbf{r}_{k}$ is a stationary process, its ensemble variance can be obtained by its temporal variance by Eq. (24), and compared with $\mathbf{P}_{k \mid k-1}^{\mathbf{Z Z}}$. When there is undue confidence that leads to a biased estimation of $\overline{\mathbf{Z}}_{k \mid k-1}$, the inconsistent $\mathbf{P}_{k \mid k-1}^{\mathbf{Z Z}}$ will be much smaller than the actual residual $\operatorname{cov}\left(\mathbf{r}_{k}\right)$

$$
\operatorname{cov}\left(\mathbf{r}_{k}\right)=E\left(\mathbf{r}_{k}^{T} \mathbf{r}_{k}\right)=\frac{1}{s} \sum_{i=1}^{s} \mathbf{r}_{i}^{T} \mathbf{r}_{i}
$$

This idea was explored by Koh and See (1994), who presented an adaptive EKF procedure by updating the process noise covariance $\mathbf{P}_{k-1}^{\mathbf{W W}}$ in time segments in order to ensure statistical consistency between $\mathbf{P}_{k \mid k-1}^{Z Z}$ and $\operatorname{cov}\left(\mathbf{r}_{k}\right)$. Along the same line, Yang et al. (2005) updated a diagonal matrix called the adaptive factor matrix, which can be regarded as a modification term of the state transfer matrix $\phi$ in Eq. (8), for a faster adaptation rate so that EKF could be used to track time-variant systems.

In a CDF context, we propose the following method to update $\hat{\mathbf{P}}_{k-1}^{\mathbf{X X}}$ if a $\operatorname{cov}\left(\mathbf{r}_{k}\right)$ is significantly larger than $\mathbf{P}_{k \mid k-1}^{\mathbf{Z Z}}$. Because the observation function Eq. (7) is usually an underdetermined system to reconstruct $\mathbf{P}_{k \mid k-1}^{\mathbf{X X}}$ given a $\mathbf{P}_{k \mid k-1}^{\mathbf{Z Z}}$ [the latter is now replaced by $\operatorname{cov}\left(\mathbf{r}_{k}\right)$ ], a least square with regularization (Tikhonov and Arsenin 1977) is employed.

Assuming addictive process and measurement noises, a simple linearization first is applied. For ease of notation, defining diagonal matrices $\mathbf{D}_{k}^{\mathbf{r}-\mathbf{V}}, \mathbf{D}_{k \mid k-1}^{\mathbf{X}}, \mathbf{D}_{k \mid k-1}^{\mathbf{X}-\mathbf{W}}$, and $\mathbf{D}_{k-1}^{\boldsymbol{\theta}}$ as

$$
\begin{gathered}
\mathbf{D}_{k}^{\mathbf{r}-\mathbf{V}} \equiv \operatorname{mdg}\left[\operatorname{cov}\left(\mathbf{r}_{k}\right)-\mathbf{P}_{k}^{\mathbf{V V}}\right] \\
\mathbf{D}_{k \mid k-1}^{\mathbf{X}} \equiv \operatorname{mdg}\left(\mathbf{P}_{k \mid k-1}^{\mathbf{X X}}\right) \\
\mathbf{D}_{k \mid k-1}^{\mathbf{X}-\mathbf{W}} \equiv \operatorname{mdg}\left(\overline{\mathbf{D}}_{k \mid k-1}^{\mathbf{X}}-\mathbf{P}_{k-1}^{\mathbf{W W}}\right) \\
\mathbf{D}_{k-1}^{\boldsymbol{\theta}} \equiv \operatorname{mdg}\left(\hat{\mathbf{P}}_{k-1}^{\boldsymbol{\theta \theta}}\right)
\end{gathered}
$$

where $\operatorname{mgg}(\mathbf{M})=$ diagonal matrix containing the main diagonal of the square matrix $\mathbf{M}$; and $\hat{\mathbf{P}}_{k-1}^{\boldsymbol{\theta \theta}}=$ submatrix in $\hat{\mathbf{P}}_{k-1}^{\mathbf{X X}}$ associating with covariance of $\boldsymbol{\theta}$, with row and column indices from $1+n_{s}$ to $n_{s}+n_{p}$. Matrices $\mathbf{J}_{k}^{\mathbf{Z} / \mathbf{X}}$ and $\mathbf{J}_{k \mid k-1}^{\mathbf{X} / \boldsymbol{\theta}}=$ approximation of the Jacobians, such defined that their $(j)$ th columns are, respectively

$$
\mathbf{J}_{k}^{\mathbf{Z} / \mathbf{X}}(j) \equiv \frac{\mathbf{Z}_{k \mid k-1}(1+j)-\mathbf{Z}_{k \mid k-1}\left(\frac{l+1}{2}+j\right)}{2 \sqrt{3 \mathbf{D}_{k \mid k-1}^{\mathbf{X}}(j, j)}} ; j=1 \text { to }\left(n_{s}+n_{p}\right)
$$

$$
\begin{gathered}
\mathbf{J}_{k \mid k-1}^{\mathbf{X} / \boldsymbol{\theta}}(j) \equiv \frac{\mathbf{X}_{k \mid k-1}\left(1+n_{s}+j\right)-\mathbf{X}_{k \mid k-1}\left(\frac{r+1}{2}+n_{s}+j\right)}{2 \sqrt{3 \mathbf{D}_{k-1}^{\boldsymbol{\theta}}(j, j)}} ; \\
j=1 \text { to } n_{p}
\end{gathered}
$$

where $\mathbf{Z}_{k \mid k-1}(i)$ is from Eq. (15); and $\mathbf{X}_{k \mid k-1}(i)$ is from Eq. (11).

By least square with regularization, one obtains $\overline{\mathbf{D}}_{k \mid k-1}^{\mathbf{X}}$ from minimization

$$
\begin{aligned}
\overline{\mathbf{D}}_{k \mid k-1}^{\mathbf{X}}= & \min _{\mathbf{\mathbf { D } _ { k | k - 1 }}}\left(\operatorname{trace}\left\{\left[\mathbf{D}_{k}^{\mathbf{r}-\mathbf{V}}-\operatorname{mdg}\left(\mathbf{J}_{k}^{\mathbf{Z} / \mathbf{X}} \mathbf{D}_{k \mid k-1}^{\mathbf{X}}\left(\mathbf{J}_{k}^{\mathbf{Z} / \mathbf{X}}\right)^{T}\right)\right]^{2}\right\}\right. \\
& \left.+\lambda \cdot \operatorname{trace}\left[\left(\mathbf{D}_{k \mid k-1}^{\mathbf{X}}\right)^{2}\right]\right)
\end{aligned}
$$

where parameter $\lambda=0.01$ is related to the regularization that tends to zero in the undetermined terms in $\overline{\mathbf{D}}_{k \mid k-1}^{\mathbf{X}}$ not constrained in Eq. (7). Comparing $\overline{\mathbf{D}}_{k \mid k-1}^{\mathbf{X}}$ with $\mathbf{D}_{k \mid k-1}^{\mathbf{X}}$, if $\overline{\mathbf{D}}_{k \mid k-1}^{\mathbf{X}}(j, j) \geqslant \mathbf{D}_{k \mid k-1}^{\mathbf{X}}(j, j)$, accept for the element in $\overline{\mathbf{D}}_{k \mid k-1}^{\mathbf{X}}$; otherwise, copy the element in $\mathbf{D}_{k \mid k-1}^{\mathbf{X}}$ to $\overline{\mathbf{D}}_{k \mid k-1}^{\mathbf{X}}$. Now, obtain $\overline{\mathbf{D}}_{k-1}^{\boldsymbol{\theta}}$ by solving the following equation in the least-square sense:

$$
\mathbf{D}_{k \mid k-1}^{\mathbf{X}-\mathbf{W}}=\operatorname{mdg}\left[\mathbf{J}_{k \mid k-1}^{\mathbf{X} / \boldsymbol{\theta}} \overline{\mathbf{D}}_{k-1}^{\boldsymbol{\theta}}\left(\mathbf{J}_{k \mid k-1}^{\mathbf{X} / \boldsymbol{\theta}}\right)^{T}\right]
$$

Compare $\overline{\mathbf{D}}_{k-1}^{\boldsymbol{\theta}}$ with $\mathbf{D}_{k-1}^{\boldsymbol{\theta}}$ and update $\hat{\mathbf{P}}_{k-1}^{\mathbf{X X}}$ using the larger elements from either of them in the corresponding position on the main diagonal of $\hat{\mathbf{P}}_{k-1}^{\mathbf{X x}}$. With this updated $\hat{\mathbf{P}}_{k-1}^{\mathbf{X X}}$, filtering in Eqs. (10)-(21) can be redone to obtain a consistent estimation of $\hat{\mathbf{X}}_{k}$ and $\hat{\mathbf{P}}_{k}^{\mathbf{X}}$.

In this study, WGI is not implemented explicitly for computational efficiency, but a similar idea is adopted; the entire recorded data are divided into segments and various a priori means are used for the filtering of different data segments. The consistency enhancement technique for the CDF outlined in Eqs. (24)-(32) is used in the two examples to be presented.

\section{Implementation}

For a structural dynamic system, inputs generally are specified in either (1) external forces on a degree of freedom (DOF), described by time histories of the external forces (a subscript $f$ will denote these DOFs and the entries in the mass, damping, and stiffness matrices that are associated with inputs of this category); or (2) a constrain trajectory on a DOF, specified by time histories of its displacement, velocity, or acceleration (a subscript $c$ will denote those DOFs and matrix entries associated with inputs of this category). Theoretically, displacement, velocity, and acceleration time histories are equivalent mutually, because given any of them one can integrate or differentiate to obtain the other two (there are practical difficulties doing so). Other DOFs are "free" in a sense that they are not subjected to prescribed time histories of external forces or trajectory constrains. However, they are brought into the first category by exerting a zero external force time history. Therefore, the structural dynamic model is described in the finite-element approach 


$$
\begin{aligned}
& {\left[\begin{array}{c:c}
M_{f f} & M_{f c} \\
\hdashline M_{c f} & M_{c c}
\end{array}\right]\left\{\begin{array}{c}
\ddot{X}_{f} \\
\hdashline \ddot{X}_{c}
\end{array}\right\}+\left[\begin{array}{c:c}
C_{f f} & C_{f c} \\
\hdashline C_{c f} & C_{c c}
\end{array}\right]\left\{\begin{array}{c}
\dot{X}_{f} \\
\hdashline \dot{X}_{c}
\end{array}\right\}+\left[\begin{array}{c:c}
K_{f f} & K_{f c} \\
\hdashline K_{c f} & K_{c c}
\end{array}\right]\left\{\begin{array}{c}
X_{f} \\
\hdashline X_{c}
\end{array}\right\}} \\
& =\left\{\begin{array}{l}
F_{f} \\
\hdashline F_{c}
\end{array}\right\}
\end{aligned}
$$

Since $\ddot{\mathbf{X}}_{c}, \dot{\mathbf{X}}_{c}$, and $\mathbf{X}_{c}$ are known by given time histories, Eq. (33) can be reduced to

$$
\mathbf{M}_{f f} \ddot{\mathbf{X}}_{f}+\mathbf{C}_{f f} \dot{\mathbf{X}}_{f}+\mathbf{K}_{f f} \mathbf{X}_{f}=\mathbf{F}_{f}-\mathbf{M}_{f c} \ddot{\mathbf{X}}_{c}-\mathbf{C}_{f c} \dot{\mathbf{X}}_{c}-\mathbf{K}_{f c} \mathbf{X}_{c}
$$

The state-space representation is then

$$
\begin{aligned}
\left\{\begin{array}{c}
\dot{\mathbf{X}}_{f} \\
\ddot{\mathbf{X}}_{f}
\end{array}\right\}= & {\left[\begin{array}{cc}
\mathbf{0} & \mathbf{I} \\
-\mathbf{M}_{f f}^{-1} \mathbf{K}_{f f} & -\mathbf{M}_{f f}^{-1} \mathbf{C}_{f f}
\end{array}\right]\left\{\begin{array}{l}
\mathbf{X}_{f} \\
\dot{\mathbf{X}}_{f}
\end{array}\right\} } \\
& +\left[\begin{array}{c}
\mathbf{0} \\
\mathbf{M}_{f f}^{-1}
\end{array}\right]\left(\mathbf{F}_{f}-\mathbf{M}_{f c} \ddot{\mathbf{X}}_{c}-\mathbf{C}_{f c} \dot{\mathbf{X}}_{c}-\mathbf{K}_{f c} \mathbf{X}_{c}\right)
\end{aligned}
$$

where $\mathbf{M}_{f f}, \mathbf{C}_{f f}, \mathbf{K}_{f f}, \mathbf{M}_{f c}, \mathbf{C}_{f c}$, and $\mathbf{K}_{f c}$ all may be parametrized by $\boldsymbol{\theta}$.

The locations where a structure is most vulnerable to damage can be narrowed based on previous experiences. It is assumed that only elements at those locations may suffer stiffness reduction, while the other elements shall have constant stiffness. Represent potential stiffness changes by stiffness correction coefficients $\beta_{i}$

$$
\beta_{i}=\frac{K_{i}^{A}}{K_{i}^{D}}
$$

where $\beta_{i}=$ stiffness correction coefficient at the $i$ th element; while $K_{i}^{A}=$ actual element stiffness; and $K_{i}^{D}=$ estimated element stiffness based on a priori knowledge, for which design documents usually are consulted to reach a starting point. Parameter $\theta$ is a collection of $\beta$ 's of a limited number of elements that are vulnerable to damage

$$
\boldsymbol{\theta}=\left\{\beta_{1}, \beta_{2}, \ldots, \beta_{l}\right\}^{T}
$$

\section{Application Examples}

Two simulation examples, one of a tower structure and the other of a bridge structure, will be presented first to demonstrate the applications of RBF for the SHM purpose in Examples 1 and 2, respectively. Then, in Example 3, a large-scale shake table test is presented to validate the proposed method.

\section{Example 1: Tower Model Subjected to Biaxial Earthquake Excitation}

In this example, a tower is modeled by two beam-column elements with two concentrated masses, as depicted in Fig. 2. Both elements are composed of the same elastic material with Young's modulus $E=28.7 \mathrm{GPa}$ and mass density $\rho=2,400 \mathrm{~kg} / \mathrm{m}^{3}$. They are of the same length $l=0.5 \mathrm{~m}$, but with different rectangular cross sections: $0.67 \times 0.8 \mathrm{~cm}^{2}$ for Element 1 and $1 \times 1.2 \mathrm{~cm}^{2}$ for Element 2. Concentrated masses are $m_{1}=0.96 \mathrm{~kg}$ and $m_{2}$ $=3.84 \mathrm{~kg}$, respectively. The tower model has its first four natural frequencies of $1.22 \mathrm{~Hz}$ (first mode along the $X$ axis), $1.46 \mathrm{~Hz}$ (first mode along the $Y$ axis), $3.67 \mathrm{~Hz}$ (second mode along the $X$

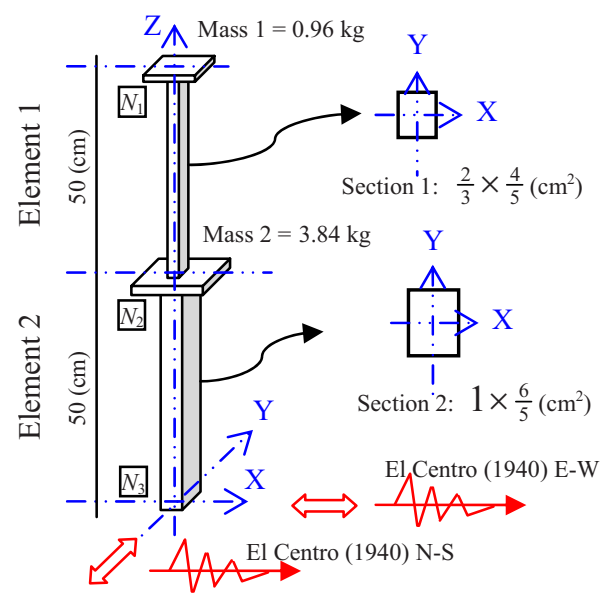

Fig. 2. Tower model subjected to biaxial earthquake excitation

axis), and $4.40 \mathrm{~Hz}$ (second mode along the $Y$ axis). Raleigh's damping is assumed with a $5 \%$ damping ratio assigned to the first two modes.

At the base (node $N_{3}$ ), the tower model is subjected to ground motions simultaneously from two directions: El Centro (1940) E-W along the $X$ axis and El Centro (1940) N-S along the $Y$ axis. The ground motion displacement, velocity, and acceleration time history records were obtained from the COSMOS Virtual Data Center (http://db.cosmos-eq.org), with displacement and velocity histories resampled at $50 \mathrm{~Hz}$; the beginning of the time histories have been tapered to zeros to eliminate the effects of the nonzero initial displacements and velocities in the records. The time histories of the acceleration records and their power spectrum density functions are plotted in Fig. 3. The tower responses to these ground motions are simulated, with velocity response at $N_{1}$ along the $X$ direction $\left(V_{1 X}\right)$ and that at $N_{2}$ along the $Y$ direction $\left(V_{2 Y}\right)$ being graphed as examples in Fig. 4.

Assume that four velocity responses are observed, i.e., $V_{1 X}$, $V_{1 Y}, \quad V_{2 X}$, and $V_{2 Y}$. Four stiffness correction coefficients, $\left\{\beta_{1}, \beta_{2}, \beta_{3}, \beta_{4}\right\}$ for the bending stiffness (represented by moments of inertia of the cross sections) $I_{1 X}$ and $I_{1 Y}$ of Element 1 , and $I_{2 X}$
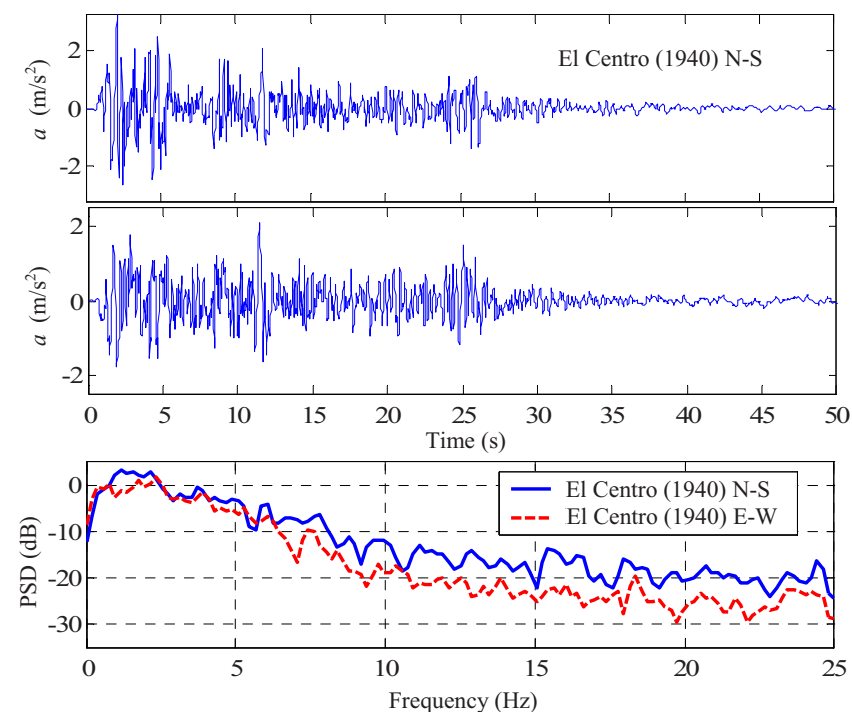

Fig. 3. Ground motions 


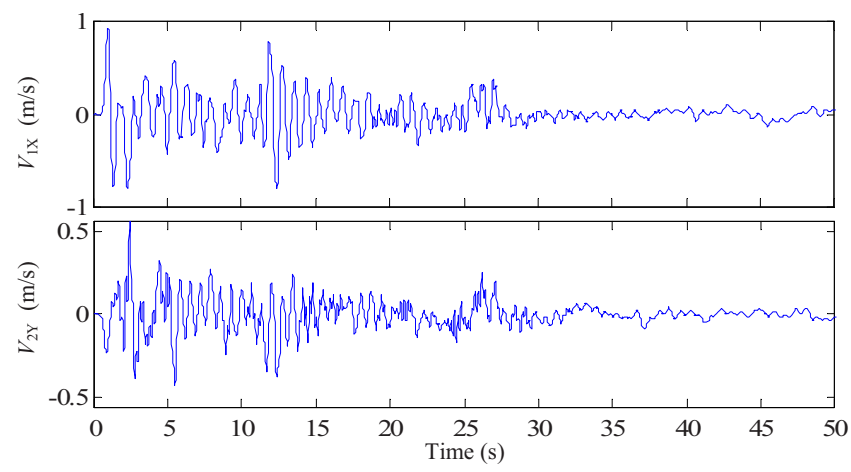

Fig. 4. Simulated tower responses
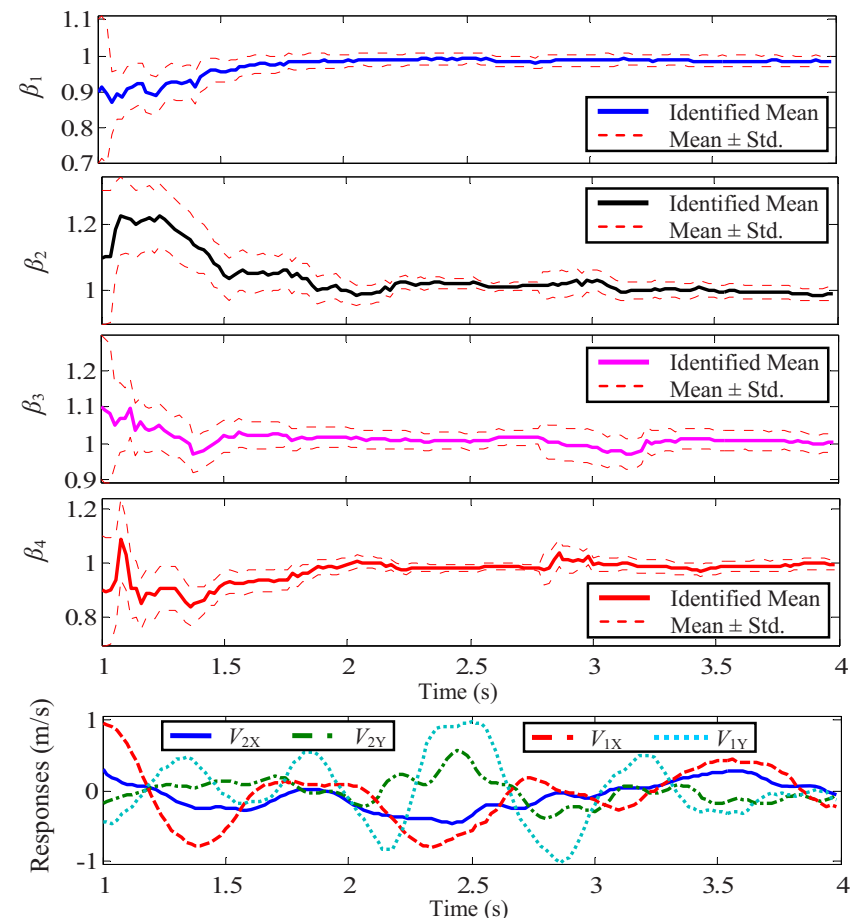

Fig. 5. Example 1: evolution of stiffness coefficients and their uncertainty

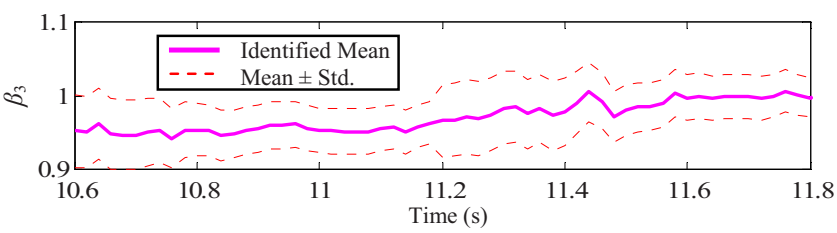

Fig. 6. Correction of inconsistent estimation

and $I_{2 Y}$ of Element 2, in both axes, respectively, are to be identified in order to assess the structural integrity of the tower model in both directions up to the element level.

Simulated responses, $V_{1 X}, V_{1 Y}, V_{2 X}$, and $V_{2 Y}$, are contaminated with white noises to form the noisy observations. The root-meansquares (RMS) of the white noises are 5\% of the RMS of the simulated responses. Similarly, noisy inputs are obtained by adding 5\% noise on top of the ground motion records. Feeding the noisy inputs and observations to the CDF implemented by Eqs. (9)-(21) with the improvement by Eqs. (24)-(32), and starting from a initial guess of element stiffness, i.e., $\left\{\beta_{1}, \beta_{2}, \beta_{3}, \beta_{4}\right\}^{\text {int }}=\{0.9,1.1,1.1,0.9\}$, the element stiffness correction coefficients $\left\{\beta_{1}, \beta_{2}, \beta_{3}, \beta_{4}\right\}$ evolute to their true values $\{1,1,1,1\}$. Meanwhile, the standard deviations of the parameters $\left\{\beta_{1}, \beta_{2}, \beta_{3}, \beta_{4}\right\}$ are gradually reduced, from the rather large a priori, $\{0.2,0.2,0.2,0.2\}$ to the much smaller a posteriori $\{0.015$, $0.019,0.026,0.020\}$, showing the reduction of their uncertainties. The evolution of the parameters and their uncertainties during a typical 3-s period $(t=1-4 \mathrm{~s})$ is graphed in Fig. 5, together with the noisy response observations during this period. This demonstrates that the mathematical model of the structure is corrected and the uncertainty associated with the model parameters is significantly diminished during this Bayesian model updating/ correction procedure, utilizing the measurements with a noise level feasible in most vibration monitoring systems.

It is observed that it requires less than 3-s measurement data for the CDF to converge to the true stiffness parameters. To test the consistency of the proposed method, nine nonoverlapping data segments, 3-s long, were taken from the earthquake record, and for each of the segments, an initial incorrect guess of $\left\{\beta_{1}, \beta_{2}, \beta_{3}, \beta_{4}\right\}^{\text {int }}$ with initial standard deviation $\{0.2,0.2,0.2,0.2\}$ was assigned to start the filtering. Each initial $\beta_{i}$ was picked randomly from a uniform distribution over interval $[0.6,1.4]$. The $\mathrm{CDF}$ results in Table 1 show that they all converged to the correct

Table 1. Example 1: Identified Element Stiffness Correction Coefficients in Nine Data Segments

\begin{tabular}{|c|c|c|c|c|c|c|c|c|c|c|c|c|}
\hline \multirow[b]{2}{*}{$\begin{array}{l}\text { Data } \\
\text { segment }\end{array}$} & \multicolumn{3}{|c|}{$\beta_{1}$} & \multicolumn{3}{|c|}{$\beta_{2}$} & \multicolumn{3}{|c|}{$\beta_{3}$} & \multicolumn{3}{|c|}{$\beta_{4}$} \\
\hline & $\begin{array}{c}\text { Initial } \\
\text { mean } \\
\text { values }\end{array}$ & $\begin{array}{c}\text { Resulting } \\
\text { mean } \\
\text { values }\end{array}$ & $\begin{array}{c}\text { Standard } \\
\text { deviation }^{\mathrm{a}}\end{array}$ & $\begin{array}{c}\text { Initial } \\
\text { mean } \\
\text { values }\end{array}$ & $\begin{array}{c}\text { Resulting } \\
\text { mean } \\
\text { values }\end{array}$ & $\begin{array}{l}\text { Standard } \\
\text { deviation }^{\text {a }}\end{array}$ & $\begin{array}{l}\text { Initial } \\
\text { mean } \\
\text { values }\end{array}$ & $\begin{array}{c}\text { Resulting } \\
\text { mean } \\
\text { values }\end{array}$ & $\begin{array}{c}\text { Standard } \\
\text { deviation }^{\mathrm{a}}\end{array}$ & $\begin{array}{l}\text { Initial } \\
\text { mean } \\
\text { values }\end{array}$ & $\begin{array}{c}\text { Resulting } \\
\text { mean } \\
\text { values }\end{array}$ & $\begin{array}{l}\text { Standard } \\
\text { deviation }\end{array}$ \\
\hline 1 & 1.391 & 1.007 & 0.016 & 0.975 & 1.016 & 0.021 & 0.652 & 0.986 & 0.018 & 0.828 & 0.977 & 0.014 \\
\hline 2 & 0.867 & 0.996 & 0.018 & 0.939 & 1.032 & 0.024 & 1.012 & 0.999 & 0.014 & 1.066 & 0.964 & 0.020 \\
\hline 3 & 1.208 & 0.985 & 0.024 & 0.781 & 1.014 & 0.024 & 1.064 & 1.005 & 0.021 & 0.946 & 0.976 & 0.024 \\
\hline 4 & 0.904 & 1.004 & 0.017 & 1.112 & 1.032 & 0.017 & 0.767 & 0.992 & 0.013 & 1.023 & 0.967 & 0.013 \\
\hline 5 & 1.054 & 0.988 & 0.020 & 1.145 & 0.976 & 0.029 & 0.969 & 1.009 & 0.021 & 1.227 & 1.004 & 0.030 \\
\hline 6 & 0.640 & 1.004 & 0.030 & 0.647 & 0.951 & 0.028 & 1.082 & 0.995 & 0.027 & 1.235 & 1.007 & 0.034 \\
\hline 7 & 0.612 & 1.024 & 0.036 & 0.844 & 1.023 & 0.028 & 1.300 & 0.953 & 0.037 & 0.932 & 1.023 & 0.033 \\
\hline 8 & 1.231 & 0.960 & 0.030 & 1.377 & 1.015 & 0.022 & 1.392 & 1.014 & 0.035 & 1.214 & 0.885 & 0.040 \\
\hline 9 & 1.115 & 1.000 & 0.035 & 0.999 & 1.030 & 0.029 & 0.771 & 1.010 & 0.031 & 0.951 & 0.953 & 0.022 \\
\hline
\end{tabular}

${ }^{\mathrm{a}}$ Resulting from the central difference filter. 
Table 2. Example 1: Identified Element Stiffness Correction Coefficients with Modeling Error

\begin{tabular}{|c|c|c|c|c|c|c|c|c|c|c|c|c|}
\hline \multirow[b]{2}{*}{$\begin{array}{l}\text { Data } \\
\text { segment }\end{array}$} & \multicolumn{3}{|c|}{$\beta_{1}$} & \multicolumn{3}{|c|}{$\beta_{2}$} & \multicolumn{3}{|c|}{$\beta_{3}$} & \multicolumn{3}{|c|}{$\beta_{4}$} \\
\hline & $\begin{array}{c}\text { Initial } \\
\text { mean } \\
\text { values }\end{array}$ & $\begin{array}{c}\text { Resulting } \\
\text { mean } \\
\text { values }\end{array}$ & $\begin{array}{l}\text { Standard } \\
\text { deviation }^{\mathrm{a}}\end{array}$ & $\begin{array}{c}\text { Initial } \\
\text { mean } \\
\text { values }\end{array}$ & $\begin{array}{c}\text { Resulting } \\
\text { mean } \\
\text { values }\end{array}$ & $\begin{array}{l}\text { Standard } \\
\text { deviation }^{\mathrm{a}}\end{array}$ & $\begin{array}{c}\text { Initial } \\
\text { mean } \\
\text { values }\end{array}$ & $\begin{array}{c}\text { Resulting } \\
\text { mean } \\
\text { values }\end{array}$ & $\begin{array}{l}\text { Standard } \\
\text { deviation }^{\mathrm{a}}\end{array}$ & $\begin{array}{c}\text { Initial } \\
\text { mean } \\
\text { values }\end{array}$ & $\begin{array}{c}\text { Resulting } \\
\text { mean } \\
\text { values }\end{array}$ & $\begin{array}{c}\text { Standard } \\
\text { deviation }^{\mathrm{a}}\end{array}$ \\
\hline 1 & 1.138 & 0.924 & 0.020 & 1.020 & 0.938 & 0.030 & 0.762 & 1.109 & 0.025 & 1.277 & 1.085 & 0.026 \\
\hline 2 & 0.904 & 0.935 & 0.015 & 0.616 & 0.922 & 0.025 & 1.145 & 1.106 & 0.015 & 1.271 & 1.104 & 0.026 \\
\hline 3 & 0.943 & 0.958 & 0.019 & 1.002 & 0.904 & 0.026 & 1.168 & 1.082 & 0.018 & 1.265 & 1.133 & 0.028 \\
\hline 4 & 1.146 & 0.976 & 0.019 & 0.752 & 0.950 & 0.024 & 0.755 & 1.066 & 0.016 & 0.844 & 1.079 & 0.026 \\
\hline 5 & 1.158 & 0.936 & 0.019 & 1.033 & 0.949 & 0.025 & 0.721 & 1.100 & 0.023 & 0.842 & 1.067 & 0.026 \\
\hline 6 & 1.075 & 0.965 & 0.031 & 1.288 & 0.877 & 0.030 & 1.283 & 1.076 & 0.029 & 0.903 & 1.084 & 0.025 \\
\hline 7 & 1.116 & 0.929 & 0.041 & 1.320 & 0.905 & 0.025 & 1.257 & 1.078 & 0.040 & 0.997 & 1.198 & 0.038 \\
\hline 8 & 0.832 & 0.873 & 0.027 & 1.128 & 0.889 & 0.018 & 0.874 & 1.111 & 0.035 & 1.254 & 1.060 & 0.045 \\
\hline 9 & 0.847 & 0.884 & 0.026 & 1.027 & 0.922 & 0.026 & 1.182 & 1.168 & 0.041 & 0.873 & 1.069 & 0.022 \\
\hline
\end{tabular}

${ }^{\mathrm{a}}$ Resulting from the central difference filter.

element stiffness, despite the various initial parameters that were far from the true values.

Note that in this example (and, also, in the next example), the consistency check by Eq. (24) was performed in every 30 data point and compared with the predicted error variance $\mathbf{P}_{k \mid k-1}^{\mathbf{Z Z}}$. If inconsistency is found, correction of the estimation confidence was performed following Eqs. (25)-(32). The filtering segment in Fig. 6 illustrates the effect of this technique devised in this study that assures consistent estimation. In this segment, $\beta_{3}$ was incorrectly identified to a value at about 0.95 in the first 30 data points, where $t \in[10.6,11.2) \mathrm{s}$, with undue confidence found by the consistency check performed at $t=11.2 \mathrm{~s}$. Although ideally, filtering of the first 30 points should be redone with the corrected a priori covariance; in order to illustrate the efficacy of the devised technique, the filtering of the first 30 points was not redone, but the corrected a priori covariance was carried to the next datum at $t=11.2 \mathrm{~s}$. It was shown that with the a priori uncertainty surges, the CDF regained its capacity to correct its estimation of the $\beta_{3}$, adapting to the new datum.

It is interesting to investigate the effect of modeling errors. Table 2 shows the results by CDF based on a model with an error, i.e., the mass at $N_{2}, m_{2}$ is assigned to $4.61 \mathrm{~kg}, 20 \%$ more than the true value. Note that since $m_{2}$ is not a parameter to be updated in $\mathrm{CDF}$, such an error cannot be corrected during filtering. However, the results in Table 2 show that CDF still converges and obtains

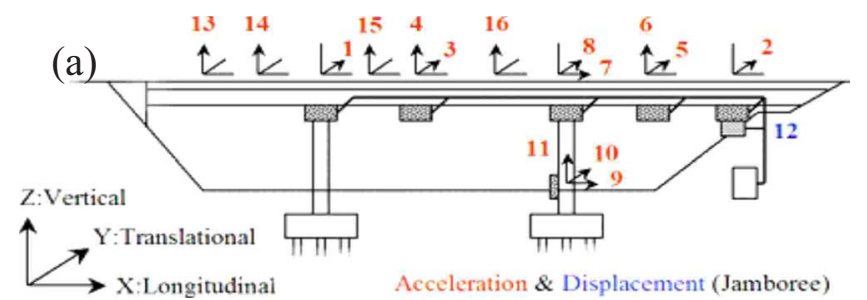

(b)

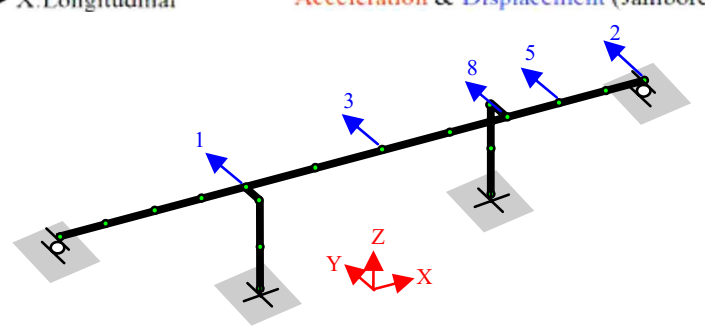

Fig. 7. Bridge model and response observations $\left\{\beta_{1}, \beta_{2}, \beta_{3}, \beta_{4}\right\}=\{0.93,0.92,1.10,1.10\}$. CDF compensates the modeling error in $m_{2}$ by identifying a stiffer Element 2 . An investigation into the modes of the resulted model (larger $m_{2}$ with stiffer Element 2) showed similarity of its modal properties to those of the true model (e.g., its natural frequencies were 122, $1.47,3.39$, and $4.09 \mathrm{~Hz}$ versus frequencies of the true model given above). This suggests that both models produce almost identical responses when subjected to same excitation. Should mass be included in the parameters to be identified together with element stiffness, such a system is ill conditioned and hard to identify because of nonuniqueness. Nonetheless, the slightly larger standard deviations associated with the error model in Table 2 compared with those in Table 1 indicated that the true model was a better fit to the observed data, and thus, more prob-

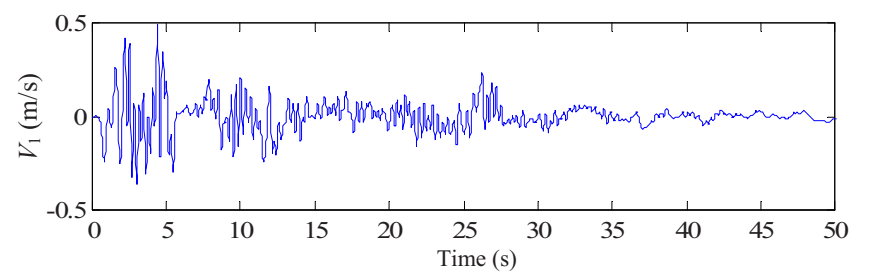

Fig. 8. Simulated bridge response at Channel 1

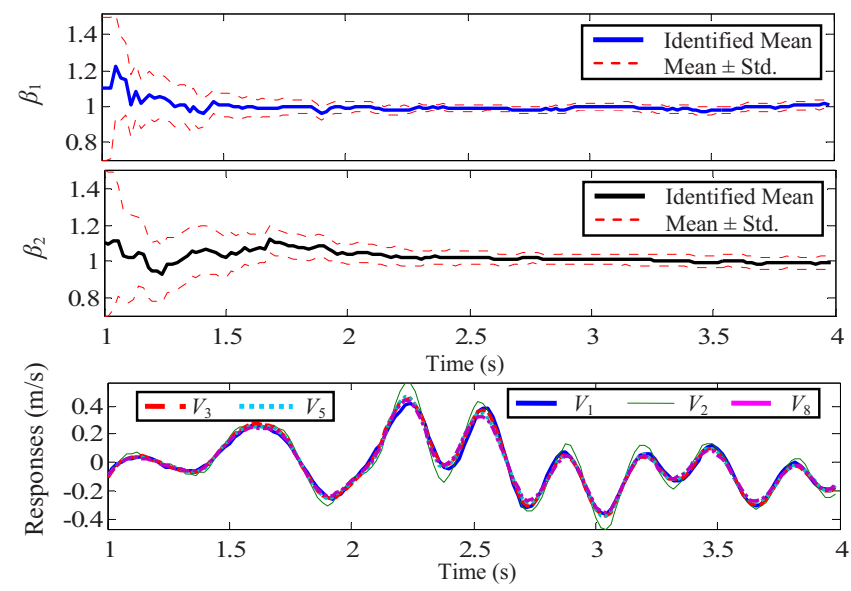

Fig. 9. Example 2: evolution of stiffness coefficients and their uncertainty 
Table 3. Example 2: Identified Stiffness Correction Coefficients in Nine Data Segments

\begin{tabular}{|c|c|c|c|c|c|c|}
\hline \multirow[b]{2}{*}{$\begin{array}{l}\text { Data } \\
\text { segment }\end{array}$} & \multicolumn{3}{|c|}{$\beta_{1}$} & \multicolumn{3}{|c|}{$\beta_{2}$} \\
\hline & $\begin{array}{c}\text { Initial } \\
\text { mean values }\end{array}$ & $\begin{array}{l}\text { Resulting } \\
\text { mean values }\end{array}$ & $\begin{array}{l}\text { Standard } \\
\text { deviation }^{\mathrm{a}}\end{array}$ & $\begin{array}{c}\text { Initial } \\
\text { mean values }\end{array}$ & $\begin{array}{l}\text { Resulting } \\
\text { mean values }\end{array}$ & $\begin{array}{l}\text { Standard } \\
\text { deviation }^{\mathrm{a}}\end{array}$ \\
\hline 1 & 1.100 & 1.012 & 0.024 & 1.100 & 0.988 & 0.035 \\
\hline 2 & 1.048 & 1.008 & 0.043 & 1.120 & 0.977 & 0.053 \\
\hline 3 & 1.172 & 0.997 & 0.023 & 1.096 & 0.995 & 0.038 \\
\hline 4 & 0.872 & 1.002 & 0.033 & 0.964 & 1.006 & 0.049 \\
\hline 5 & 1.176 & 1.023 & 0.039 & 1.168 & 1.002 & 0.065 \\
\hline 6 & 0.968 & 1.004 & 0.042 & 1.160 & 0.969 & 0.071 \\
\hline 7 & 0.824 & 0.972 & 0.035 & 0.944 & 1.027 & 0.058 \\
\hline 8 & 1.128 & 0.997 & 0.031 & 0.804 & 1.022 & 0.057 \\
\hline 9 & 0.856 & 1.006 & 0.035 & 0.884 & 0.973 & 0.052 \\
\hline
\end{tabular}

${ }^{\mathrm{a}}$ Resulting from the central difference filter.

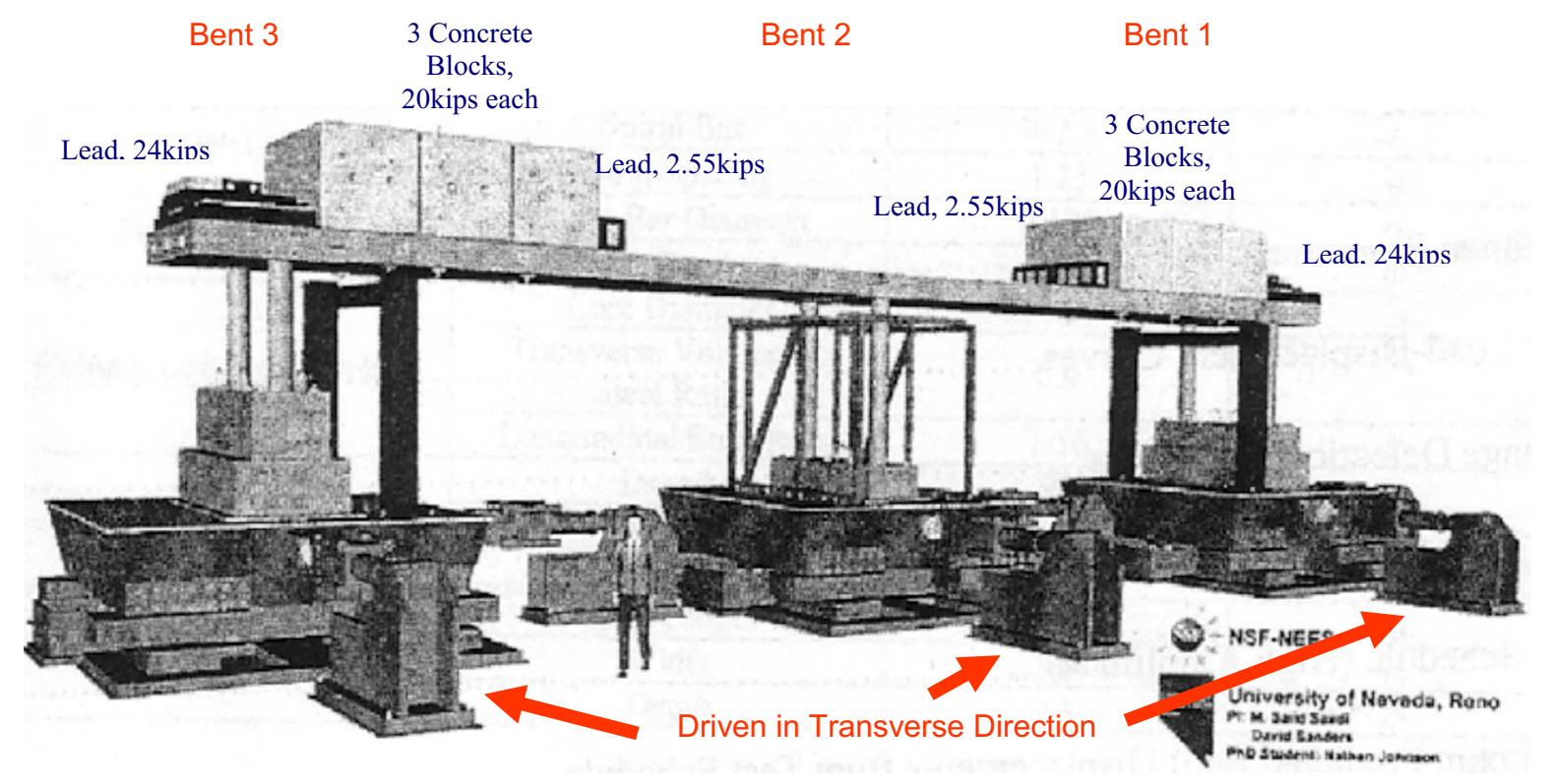

\section{(a) 2-span 3-bent bridge specimen}

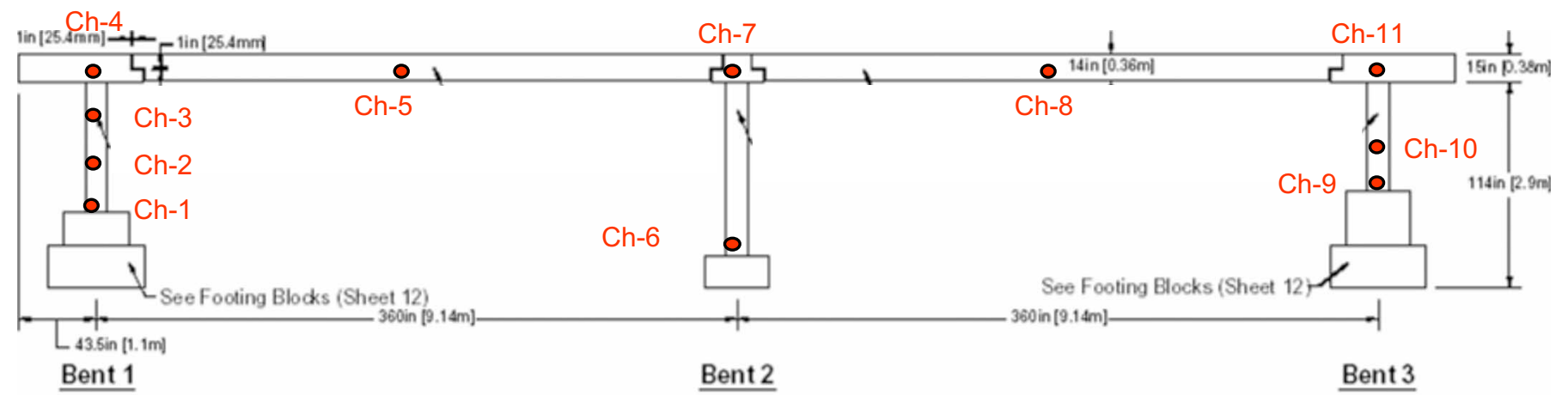

Elevation View

\section{(b) Sensor layout}

Fig. 10. Large-scale shake table test setup 
able. How to assure the identifiability of a system is an important issue, it is related to the parametrization, sensor number, location selection, and the choice of excitation sources. Thorough discussion on this issue is beyond the scope of this paper.

Example 1 demonstrates that the proposed CDF algorithm can correctly identify the bending stiffness of each individual element in both directions, and can obtain a consistent estimation of the uncertainty of the stiffness coefficients. Therefore, this method can detect stiffness changes to the element level. It also is noted that in this example, filtering of each of the short segments is initiated randomly, blind to previous and later data or the system status. This indicates the rapid convergence of CDF: data of very short length, a few seconds in this case (only a couple of cycles in terms of the system fundamental period) are sufficient for the $\mathrm{CDF}$ algorithm to reach a meaningful identification. This is a significant advantage over most existing parameter identification methods. This feature is desirable especially in SHM applications. When any element stiffness steps down in a damaging event, applying CDF to very short data segments recorded before and after the event will reveal the structure health condition change (including damage location and intensity). Although the intent is not to trace the instantaneous stiffness during a highly nonlinear event when a system experiences complicated hysteresis loops, the proposed method is able to keep track of degradation of a structure when its stiffness changes are not faster than its fundamental frequency. This method also is good for postevent structural condition evaluation when a structure assumes a decreased but stable stiffness.

\section{Example 2: Bridge Model Subjected to Earthquake Excitation}

This example imitates the situation of a typical highway bridge instrumented for longterm health monitoring purposes. The prototype bridge in Fig. 7(a) is a three-span continuous cast-in-place posttensioned box-girder bridge. The total length of the bridge was $110.9 \mathrm{~m}$ with each span length of $35.5,46.1$, and $30.3 \mathrm{~m}$. The bridge was straight and supported on two monolithic single columns and sliding bearings on both abutments. There were offsets from the centers of the columns to the central line of the superstructure. It was instrumented with 16 vibration sensors. During earthquakes, the major concern was the structural integrity in the transverse $(Y)$ direction, therefore, only the vibration measurements in the $Y$ direction (Channels 1, 2, 3, 5, and 8, as shown in Fig. 7) were considered as the observations. It was assumed that the ground motion time histories were identical at the bases of both columns, and could be recorded by the sensors at the footing (i.e., Channel 10 in this case). The finite-element model of this bridge is shown in Fig. 7(b).

To evaluate the integrity conditions of the super- and substructures, respectively, two parameters were to be identified by the CDF: $\left\{\beta_{1}, \beta_{2}\right\}$, where $\beta_{1}=$ stiffness correction coefficient of the transverse bending (about the $Z$ axis) for all the elements of the box-girder deck; and $\beta_{2}=$ stiffness correction coefficient of the transverse bending (about the $X$ axis) for all the elements of the two columns. To illustrate the capacity of the CDF method, bridge responses to the El Centro (1940) N-S earthquake (tapered as in Fig. 3) along the $Y$ axis first were simulated with $\left\{\beta_{1}, \beta_{2}\right\}$ set to $\{1,1\}$. The simulated velocity response at Channel 1 is graphed in Fig. 8.

Similar to Example 1, earthquake records and the simulated responses were contaminated with $5 \%$ noise to form the noisy inputs and the noisy observations, which were fed into the CDF to
Table 4. Test Procedure

\begin{tabular}{lccc}
\hline Tests & $\begin{array}{c}\text { Ground motion } \\
\text { description } \\
\text { (in transverse) }\end{array}$ & $\begin{array}{c}\text { Peak } \\
\text { ground } \\
\text { acceleration }\end{array}$ & $\begin{array}{c}\text { Damage } \\
\text { description }\end{array}$ \\
\hline WN-X-1 & White noise & & \\
T-12 & Low earthquake & 0.085 & Bent 1 yields \\
T-13 & Low earthquake & 0.173 & Bent 3 yields \\
T-14 & Moderate earthquake & 0.319 & Bent 2 yields \\
WN-X-2 & White noise & & \\
T-15 & High earthquake & 0.627 & \\
T-16 & Severe earthquake & NA & \\
T-17 & Extreme earthquake & 1.14 & Bent 3 steel \\
WN-X-3 & White noise & & buckles \\
T-18 & Extreme earthquake & 1.398 & \\
T-19 & Extreme earthquake & 1.703 & \\
& & & \\
WN-X-4 & White noise & 1.286 & \\
T-20 & After shot & & \\
WN-X-5 & White noise & &
\end{tabular}

Note: NA=not available.

update the structural model. Nine nonoverlapping data segments, each with a 3-s duration, were taken from the earthquake record. For each of the data segments, random initial guesses of $\beta_{1}$ and $\beta_{2}$ were picked independently from a uniform distribution over the interval $[0.8,1.2]$, with their initial standard deviation assigned to $\{0.4,0.4\}$. Despite the random starting values, $\left\{\beta_{1}, \beta_{2}\right\}$ converged to the true values $\{1,1\}$ in all the filtering using different data segments (Table 3). The evolution of estimation of stiffness coefficients and their uncertainties during the first data segment $(t=1-4 \mathrm{~s})$ is graphed in Fig. 9.

\section{Example 3: Large-Scale Shake Table Test of a Two-Span Three-Bent Bridge Model}

A series of large-scale shake table tests were conducted at the University of Nevada, Reno (UNR), in a National Science Foundation-Network for Earthquake Engineering Simulation (NSF-NEES) sponsored research to explore the seismic behaviors of reinforced concrete bridges (Johnson et al. 2006). Taking advantage of this opportunity, the writers collaborated with researchers at UNR and installed additional accelerometers on the bridge specimen to verify the proposed methodology of structural condition assessment for bridge bents using pre- and postevent vibration data.

Shake table tests were performed on a two-span three-bent reinforced concrete bridge specimen, shown in Fig. 10(a). Each of the three bents was supported on an individual shake table. The bents were linked by the bridge deck, with a total length of $18.29 \mathrm{~m}$ (720 in.). Each of the bents consisted of two columns, having the same design cross sections with a diameter of $0.3 \mathrm{~m}$ (12 in.). The bents were of different heights, $1.83 \mathrm{~m}$ (72 in.), $2.44 \mathrm{~m}$ (96 in.), and $1.52 \mathrm{~m}$ (60 in.) for Bents 1, 2, and 3, respectively, so that they processed significantly different transverse stiffnesses. To resemble the inertia of other parts of the superstructure not built into this specimen, compensative masses were added. The shake tables were driven by input acceleration signals in the transverse direction of the bridge. Eleven accelerometers were installed on the specimen to obtain the acceleration inputs and responses of the bridge, as illustrated in Fig. 10(b).

During the tests, earthquake ground motions of increasing in- 
Table 5. Example 3: Identified Stiffness Correction Coefficients

\begin{tabular}{|c|c|c|c|c|c|c|c|c|c|}
\hline \multirow[b]{2}{*}{$\begin{array}{l}\text { Data } \\
\text { (test) }\end{array}$} & \multicolumn{3}{|c|}{$\beta_{1}$} & \multicolumn{3}{|c|}{$\beta_{2}$} & \multicolumn{3}{|c|}{$\beta_{3}$} \\
\hline & $\begin{array}{c}\text { Initial } \\
\text { mean values }\end{array}$ & $\begin{array}{l}\text { Resulting } \\
\text { mean values }\end{array}$ & $\begin{array}{c}\text { Standard } \\
\text { derivation }^{\text {a }}\end{array}$ & $\begin{array}{c}\text { Initial } \\
\text { mean values }\end{array}$ & $\begin{array}{l}\text { Resulting } \\
\text { mean values }\end{array}$ & $\begin{array}{c}\text { Standard } \\
\text { deviation }^{\text {a }}\end{array}$ & $\begin{array}{c}\text { Initial } \\
\text { mean values }\end{array}$ & $\begin{array}{l}\text { Resulting } \\
\text { mean values }\end{array}$ & $\begin{array}{l}\text { Stnadard } \\
\text { deviation }^{\mathrm{a}}\end{array}$ \\
\hline $\mathrm{X}-\mathrm{WN}-1$ & 1.00 & 0.754 & 0.023 & 1.00 & 0.747 & 0.071 & 1.00 & 0.758 & 0.030 \\
\hline $\mathrm{X}-\mathrm{WN}-2$ & 0.75 & 0.512 & 0.014 & 0.75 & 0.748 & 0.058 & 0.75 & 0.563 & 0.012 \\
\hline X-WN-3 & 0.50 & 0.190 & 0.004 & 0.70 & 0.286 & 0.027 & 0.56 & 0.165 & 0.005 \\
\hline $\mathrm{X}-\mathrm{WN}-4$ & 0.15 & 0.175 & 0.006 & 0.50 & 0.200 & 0.047 & 0.12 & 0.095 & 0.006 \\
\hline X-WN-5 & 0.17 & 0.169 & 0.004 & 0.13 & 0.172 & 0.020 & 0.12 & 0.102 & 0.005 \\
\hline
\end{tabular}

${ }^{\mathrm{a}}$ Resulting from the central difference filter.

tensity, from low, moderate, high, severe, and extreme, were used as the driving signals of the shake tables to simulation of strong motions. After the strongest ground motion, a smaller motion was input to mimic an after-shot earthquake. In Table 4, the sequence of the strong motions [denoted by "T-(number)"] and their input peak ground accelerations (PGA) are listed. Different levels of damage were introduced to the bridge specimen by these strong motions. In between the strong motions, low-amplitude white noise [denoted by "WN-X-(number)"] with a PGA of approximately $0.05 g$, drove the shake tables to perturb the specimen in the corresponding damage level. Such perturbations did not introduce further nonlinearity and the system under a perturbation behaved as a linear system with a stable stiffness.

As indicated in Table 4, the damage procedure observed can be outlined as: Bent 1 yields $\rightarrow$ Bent 3 yields $\rightarrow$ Bent 2 yields $\rightarrow$ Bent 3 steel buckles. This procedure is largely determined by the relative heights of the bents. The onset of Bent 1 yielding is due to the fact that the first mode of this bridge specimen (in its undamaged stage) has the largest displacement on Bent 1. After the yielding of Bent 1, Bent 3 attracts most of the seismic force and yields, and then so happens to Bent 2 after the yielding of Bents 1 and 3. The final collapse is associated with the steel buckling at Bent 3, which has the smallest ductility capacity among the three.

Acceleration measurements at bent footings are considered as inputs (Ch-1, Ch-6, and Ch-9), while those on the superstructure (Ch-4, Ch-5, Ch-7, Ch-8, and Ch-11) are system outputs. Data obtained in low-amplitude white-noise vibrations WN-X-1-WN$\mathrm{X}-5$ were fed into the CDF described above to identify the outof-plan bending stiffness of each bent. Identification results are tabulated in Table 5. Note that the identified sectional stiffness coefficients clearly indicate the same damage procedure as observed in the tests. Between WN-X-1 and WN-X-2, $\beta_{1}$ and $\beta_{3}$ dropped from 0.75 to 0.51 and from 0.76 to 0.56 , respectively, while $\beta_{2}$ remained at the same level, indicating in a quantitative manner the yielding of Bents 1 and 3 between these two tests. Then, between WN-X-2 and WN-X-3, the decrements in all, $\beta_{1}$, $\beta_{2}$, and $\beta_{3}$ signal that not only Bent 2 yielded, but also the damage in Bents 1 and 3 further developed. In WN-X-4, $\beta_{3}$ touched down to a very low value, 0.10 , associated with the severe damage in Bent 3 (steel buckling). And the results of $\mathrm{WN}-\mathrm{X}-5$ are comparable to those in $\mathrm{WN}-\mathrm{X}-4$, which is consistent with the observation that the after-shot earthquake actually had not further damaged the bridge specimen significantly.

The identified bent stiffness changes also were found to be consistent with those extracted from the experimental hysteresis curves recorded by the researchers at UNR (Johnson et al. 2006). The UNR data were downloaded from the NEES database and replotted by Chen et al. (2008).

\section{Conclusion}

This paper presents a new vision of SHM in a Bayesian point of view, where SHM is a procedure of information collection that corrects and updates the probabilistic mathematical model of a structure, and gradually diminishes the modeling uncertainty. The $\mathrm{RBF}$ implemented by CDF, a superior algorithm over the conventional EKF because of its utilizing a high-fidelity propagation of a probability density in a nonlinear system, is applied in this research in conjunction with a consistency enhancement technique to achieve the redefined goal of SHM. Two numerical examples, one of a tower model and one of a bridge structure, are presented to illustrate the capacities of the CDF algorithm to correctly and consistently estimate the model parameters and their uncertainties. Data from a large-scale shake table test on a reinforced concrete bridge specimen validate the proposed method. The dataupdated probabilistic models (represented by parameters and their uncertainties) of the structures are indicators of the structural integrity conditions, and useful for evaluating the current or future structural performances in a probabilistic sense.

The proposed CDF algorithm requires both excitation and response measurements. The Bayesian method, however, is general and can adapt easily to output-only situations, if the system excitation is described adequately in a statistical model. The proposed CDF algorithm converged rapidly. Data of only a couple of cycles in terms of the system fundamental period were sufficient for it to reach a meaningful identification. This significant advantage is especially desirable in SHM applications, which enables a SHM system to keep track of degradation of a structure when the rate of structural status variation is slightly lower than its fundamental frequency. This method also is suitable for postevent structural condition evaluation when a structure assumes a decreased but stable stiffness compared with the preevent condition.

\section{Acknowledgments}

This research is supported by Caltrans, under Contract No. 59A0311, and managed by Mr. Li-Hong Sheng.

\section{References}

Beck, J. L. (1989). "Statistical system identification of structures." Proc., 5th Int. Conf. on Structural Safety and Reliability, A. H.-S. Ang, M. Shinozuka, and G. I. Shuëller, eds., ASCE, New York, 1395-1402.

Beck, J. L., and Au, S.-K. (2002). "Bayesian updating of structural models and reliability using Markov chain Monte Carlo simulation." J. Eng. Mech., 128(4), 380-391. 
Beck, J. L., and Katafygiotis, L. S. (1998). "Updating models and their uncertainties. I: Bayesian statistical framework." J. Eng. Mech., 124(4), 445-461.

Beck, J. L., and Yuan, K.-V. (2004). "Model selection using response measurements: Bayesian probabilistic approach." J. Eng. Mech., 130(2), 192-203.

Bolton, R., Stubbs, N., Park, S., and Choi, S. (2001). "Documentation of changes in modal properties of a concrete box-girder bridge due to environmental and internal conditions." Comput. Aided Civ. Infrastruct. Eng., 16, 42-57.

Chen, Y., Feng, M. Q., and Soyoz, S. (2008). "Large-scale shake table test verification of bridge condition assessment methods." J. Struct. Eng., 134(7), 1235-1245.

Chui, C. K., and Chen, G. (1991). Kalman filtering: With real-time applications, Springer, New York.

Doebling, S. W., Farrar, C. R., Prime, M. B., and Shevitz, D. W. (1996). "Damage identification and health monitoring of structural and mechanical systems from changes in their vibration characteristics: A literature review." Los Alamos National Laboratory Rep. LA-13070$M S$, Los Alamos National Laboratory, Los Alamos, N.M.

Ghanem, R., and Shinozuka, M. (1995). "Structural-system identification. I: Theory." J. Eng. Mech., 121(2), 255-264.

Grewal, M. S., and Andrews, A. P. (2001). Kalman filtering: Theory and practice using MATLAB, 2nd Ed., Wiley, New York.

Hera, A. (2004). "Application of wavelet approach for ASCE structural health monitoring benchmark studies." J. Eng. Mech., 130(1), 96104.

Hoshiya, M., and Saito, E. (1984). "Structural identification by extended Kalman filter." J. Eng. Mech., 110(12), 1757-1770.

Hoshiya, M., and Sutoh, A. (1993). "Kalman filter-finite-element method in identification." J. Eng. Mech., 119(2), 197-210.

Johnson, N. S., Saiidi, M., and Sanders, D. H. (2006). "Large-scale experimental and analytical seismic studies of a two-span reinforced concrete bridge system." Rep. No. CCEER-06-02, Center for Civil Engineering Earthquake Research, Dept. of Civil and Environmental Engineering, Univ. of Nevada, Reno, Nev.

Koh, C. G., Qiao, G. Q., and Quek, S. T. (2003). "Damage identification of structural members: Numerical and experimental studies." Struct. Health Monit., 2(1), 41-55.

Koh, C. G., and See, L. M. (1994). "Identification and uncertainty estimation of structural parameters." J. Eng. Mech., 120(6), 1219-1236.

Lam, H. F., Katafygiotis, L. S., and Mickleborough, N. D. (2004). "Application of a statistical model updating approach on Phase I of the IASE-ASCE structural health monitoring benchmark study." J. Eng. Mech., 130(1), 34-48.

Moradkhani, H., Sorooshian, S., Gupta, H. V., and Houser, P. R. (2005). "Dual state-parameter estimation of hydrological models using ensenble Kalman filter." Adv. Water Resour., 28, 135-147.

Oreta, A. W. C., and Tanabe, T. (1994). "Element identification of member properties of framed structures." J. Struct. Eng., 120(7), 1961-1976.

Papadimitriou, C., Beck, J. L., and Katafygiotis, L. S. (2001). "Updataing robust reliability using structural test data." Probab. Eng. Mech., 16, 103-113.

Shinozuka, M., and Ghanem, R. (1995). "Structural system identification. II: Experimental verification.” J. Eng. Mech., 121(2), 265-273.

Shinozuka, M., Yun, C.-B., and Imai, H. (1982). "Identification of linear structural dynamic systems.” J. Engrg. Mech. Div., 108(6), 13711390.

Sohn, H., Farrar, C. R., Hemez, F. M., Shunk, D. D., Stinemates, D. W., and Nadler, B. R. (2003). "A review of structural health monitoring literature: 1996-2001." Los Alamos National Laboratory Rep. LA13976-MS, Los Alamos National Laboratory, Los Alamos, N.M.

Srinivasan, R. (2002). Importance sampling: Applications in communications and detection, Springer, Berlin.

Tikhonov, A. N., and Arsenin, V. Y. (1977). Solution of ill-posed problems, Wiley, New York.

Van der Merwe, R. (2004). "Sigma-point Kalman filters for probabilistic inference in dynamic state-space models." Ph.D. dissertation, Oregon Health and Science Univ., Portland, Ore.

Vanik, M. W., Beck, J. L., and Au, S. K. (2000). "Bayesian probabilistic approach to structural health monitoring.” J. Eng. Mech., 126(7), 738-745.

Wan, E., and van der Merwe, R. (2001). "The unscented Kalman filter." Kalman filtering and neural networks, S. Haykin, ed., Wiley, New York.

Yang, J. N., and Lin, S. (2005). "Identification of parameter variations of structures based on least squares estimation and adaptive tracking technique.” J. Eng. Mech., 131(3), 290-298.

Yang, J. N., Lin, S., Huang, H., and Zhou, L. (2005). "An adaptive extended Kalman filter for structural damage identification." Struct. Control Health Monit., 13(4), 849-867.

Yoshida, I., and Sato, T. (2002a). "Health monitoring algorithm by the Monte Carlo filter based on non-Gaussian noise." J. Nat. Disaster Sci., 24(2), 101-107.

Yoshida, I., and Sato, T. (2002b). "Identification of damping ratio using Monte Carlo filter based on exclusive non-Gaussian process noise." Proc., 15th Engineering Mechanics Conf., ASCE, Reston, Va.

Yuen, K.-V., Beck, J. L., and Au, S. K. (2004). "Structural damage detection and assessment by adaptive Markov chain Monte Carlo simulation." Struct. Control Health Monit., 11, 327-347.

Yun, C.-B., Kim, W. J., and Ang, A. H.-S. (1989). "Damage assessment of bridge structures by system identification." Proc., 5th Int. Conf. on Structural Safety and Reliability, A. H.-S. Ang, M. Shinozuka, and G. I. Shuëller, eds., ASCE, New York.

Yun, C.-B., and Shinozuka, M. (1980). "Identification of nonlinear structural dynamic systems.” J. Struct. Mech., 8(2), 187-203. 